\title{
Cómo mejorar la limpieza colónica
}

\author{
Raquel Palos-Cuéllar* \\ Hospital General de Zona N. ${ }^{\circ} 89$, Instituto Mexicano del Seguro Social, Guadalajara, Jal., México
}

La limpieza intestinal es clave para lograr una colonoscopia de calidad, es por esto que sigue siendo un área que se investiga activamente.

\section{Tipos de preparación intestinal}

Existe un nuevo medicamento, NER1006 (Plenvu $\left.{ }^{\circledR}\right)$, que fue aprobado en 2018 en EE.UU. para ser utilizado en la preparación intestinal en adultos, pero en pacientes con disminución en la función renal no ha sido evaluado. Un estudio con más de 700 pacientes ${ }^{1}$ investigó el perfil de seguridad de NER1006 (Plenvu ${ }^{\circledR}$, Salix Pharmaceuticals) 1 I PEG (polietilenglicol) como preparación intestinal en pacientes con IR (insuficiencia renal) (aclaramiento de creatinina; IR leve: $>60$ a $<90 \mathrm{ml} / \mathrm{min}$, IR moderada: $<60 \mathrm{ml} / \mathrm{min}$ ). Los efectos adversos más comúnmente observados fueron gastrointestinales: náuseas y vómitos (hasta en el $14 \%$ de los pacientes) pero esto no condicionó intolerancia a la preparación. Solo en un paciente con insuficiencia renal moderada se encontró elevación leve de creatinina $>0.3 \mathrm{mg} / \mathrm{dl}$ que no persistió más de 7 días. Concluyen que esta preparación intestinal es segura en pacientes con insuficiencia renal de leve-moderada ${ }^{1}$. Otro estudio comparó la preparación intestinal con Plenvu $^{\circledR}$ vs. PEG 21 + ácido ascórbico². Evaluaron el éxito en la limpieza colónica completa (grupo Plenvu ${ }^{\circledR}$ puntuación Boston $7.83 \pm 1.45$ vs. grupo PEG 2 | $7.58 \pm$ $1.40 p=0.068$ ), y por segmentos (porcentaje de pacientes con un puntaje mayor a 2, Plenvu ${ }^{\circledR}$ vs. PEG $21+$ ácido ascórbico, respectivamente $=$ colon derecho 95.4 vs. 94.19 [p 0.43], colon transverso 97.13 vs. 98.26 [ $p=0.15]$, colon izquierdo 96.55 vs. 97.09) [ $p=0.15]$, el índice de detección de pólipos fue significativamente mayor en el grupo de Plenvu ${ }^{\circledR}$ que en el grupo PEG 2 I + ácido ascórbico (48.85 vs. $37.39 \% ; p<0.0001$ ), índice de detección de adenomas fue numéricamente más alto en el grupo Plenvu ${ }^{\circledR}$ vs. grupo PEG 2 I + ácido ascórbico (24.71 vs. $20.35 \% ; p=0.3314$ ). Los efectos adversos que se presentaron con ambas preparaciones intestinales fueron principalmente gastrointestinales (distensión abdominal, dolor abdominal, náuseas y vómitos) y de sistema nervioso (mareos). Concluyendo que Plenvu ${ }^{\circledR}$ fue tan efectivo como PEG 2 I + ácido ascórbico en la limpieza colónica total, con mejoría del índice de detección de pólipos ${ }^{2}$.

\section{Educación}

Existen múltiples estudios que han demostrado que la educación del paciente es muy importante para una adecuada preparación intestinal. Un estudio realizado en 593 pacientes $^{3}$, demostró que al tener acceso a un sitio web donde se les proporcionó información, vídeos, explicaciones, etc., previo a la realización de la colonoscopia impactó de forma positiva, ya que aquellos que visitaron el sitio web consumieron adecuadamente la dosis dividida de la preparación comparado con los que no visitaron el sitio web (68.2 vs. $52.5 \% p=0.0062$ ). En aquellos que tomaron la preparación dividida hubo mejor puntuación de Boston (9 vs. 7; $p<0.0001$ ). El $69.7 \%$ de los pacientes que visitaron el sitio web tuvieron reducción de la ansiedad-estrés relacionado con el procedimiento. Concluyendo que el uso de una plataforma informativa en línea puede ayudar a mejorar la educación del paciente previo a la realización de la colonoscopia, reduciendo la ansiedad y estrés que están asociados al

\section{Correspondencia:}

*Raquel Palos-Cuéllar

E-mail: dra.raquelpaloscuellar@gmail.com

DOI: 10.24875/END.M21000334

Endoscopia. 2021;33(Supl 1):13-15 0188-9893/๑ 2021. Asociación Mexicana de Endoscopia Gastrointestinal, publicado por Permanyer México SA de CV, todos los derechos reservados. 
procedimiento, mejora el apego a la preparación intestinal y mejora la puntuación de limpieza intestinal.

\section{Obesidad}

En un estudio ${ }^{4}$ en el que se incluyeron más de 27,000 pacientes, que tenía la intención de identificar los efectos del índice de masa corporal en el éxito, eficacia y tolerabilidad de la colonoscopia, se demostró que en los pacientes que presentan obesidad o sobrepeso fue más probable una colonoscopia incompleta $(p<0.05)$, un procedimiento más largo ( $>30$ minutos en pacientes con sobrepeso u obesidad, $p<0.001)$, una preparación intestinal inadecuada (más prevalente en el cohorte de obesidad grado III, $p<0.001$ ) y pobre tolerancia durante el procedimiento ( $18.7 \%$ colonoscopia incompleta por poca tolerancia del paciente y fue significativamente mayor en el grupo de sobrepeso y obesidad, $p<0.001)^{4}$.

\section{Evaluación adecuada del colon}

La presencia de burbujas intraluminales durante la colonoscopia puede disminuir la adecuada visualización de la mucosa colónica, lo cual puede llevar a una disminución en el índice de detección de adenomas y prolongar el tiempo del procedimiento. En un estudio se determinaron los factores que están asociados a la formación de burbujas durante la colonoscopia ${ }^{5}$. Encontraron que edad más joven (OR: 0.98; IC 95\%: 0.96-0.99) y no beber la preparación colónica fría (OR: 0.63; IC 95\%: 0.41-0.98) fueron los únicos dos factores independientemente asociados con la presencia de burbujas durante la colonoscopia 5 .

\section{Mala preparación intestinal}

Cuando la preparación colónica es inadecuada, las guías de tratamiento ASGE recomiendan una colonoscopia de seguimiento en 1 año ${ }^{6}$. No existen suficientes datos sobre el cumplimiento de la colonoscopia al año de seguimiento. Considerando mala preparación intestinal una escala de Boston $<6$ se realizó un estudio en el que analizaron el porcentaje de pacientes que volvieron para realizarse su colonoscopia al año de seguimiento. De los 345 pacientes a los que se les recomendó regresar al año de seguimiento solo el $29.6 \%$ lo hizo, $19 \%$ regreso entre 1-2 años, y $51.3 \%$ no regresó. El $35 \%$ no regresaron debido a muerte, edad avanzada, problemas con su seguro medico, preocupación por COVID-19, y otros problemas médicos. De los pacientes que repitieron su colonoscopia el $81.4 \%$ tuvieron una adecuada preparación, y un mayor número de adenomas fueron detectados comparados con la colonoscopia índice (134 vs. $77 ; p=0.0043$ ). La preparación colónica inadecuada está asociada con peores resultados clínicos: incremento en el índice de complicaciones, lesiones patológicas pérdidas, etc. Aproximadamente el $30-50 \%$ de los pacientes hospitalizados que se someten a una colonoscopia tienen una limpieza colónica inadecuada. ${ }^{7}$ En un estudio decidieron evaluar el impacto de las preparaciones de bajo volumen vs. las preparaciones de alto volumen ${ }^{7}$. Fue un estudio retrospectivo, observacional, en un solo centro, incluyó a 1,807 pacientes; las preparaciones de bajo volumen estuvieron asociadas con disminución en el tiempo (días) de la preparación a la realización de la colonoscopia (1.1 vs. $1.5 ; p<0.001$ ), por lo cual disminuyó también la estancia hospitalaria y costos; es mejor tolerada y la calidad de la preparación en comparable con las preparaciones de alto volumen?.

\section{Desempeño del endoscopista}

La calidad de la colonoscopia también puede estar influenciada por la fatiga del operador ${ }^{8}$. Un estudio investigó si realizar la colonoscopia al final de la semana modifica la calidad de esta. Se estudiaron más de 45,000 colonoscopias consecutivas, encontrando en sus resultados que el índice de detección de adenomas (RR: 1.01; IC 95\%: 0.88-1.15; $p=0.94$ ), el índice de deteccion de pólipos serrados sésiles (RR: 0.90; IC 95\%: 0.70-1.14; $p=0.38$ ), índice de detección de pólipos (RR: 1.00; IC 95\%: 0.92-1.09; $p=0.94$ ) y falla en la intubación cecal (RR: 0.92; IC 95\%: 0.721.18; $p=0.51$, no son estadísticamente diferentes el final de la semana comparado con el resto de la semana ${ }^{8}$. Hay otro estudio que también busco determinar si la fatiga de la semana de trabajo puede contribuir con el desempeño del endoscopista ${ }^{9}$. Fue un estudio retrospectivo, que incluyó más de 2,000 pacientes, encontrando que el índice de detección de adenomas fue significativamente menor en viernes que en el resto de la semana $(34.9 \pm 2.3 \% ; p<0.008)$. Adenoma por colonoscopia $(p<0.006)$, así como índice de detección de pólipos $(p<0.029)$ en viernes fue significativamente menor que el resto de la semana. Adenoma por participante positivo en viernes $(1.64 \pm 1)$ fue significativamente menor que el resto de la semana a excepción del miércoles $(1.8 \pm 0.1$; $p<0.005$ y $p=0.224$ respectivamente). Índice de detección de pólipos en colon derecho en viernes 
$(32.8 \pm 2.3 \%)$ fue significativamente menor que todos los demás días de la semana a excepción del miércoles $(38.1 \pm 2.4 \% ; p<0.003$ y $p=0.124$, respectivamente). El tiempo de retirada en viernes fue significativamente menor que los demás días de la semana (17.2 minutos $\pm 0.61 ; p<0.001)$. Concluyendo que para las colonoscopias de escrutinio hay una disminución en el desempeño del endoscopista en viernes cuando es comparado con otros días de la semana. Estos hallazgos indican un componente potencial de fatiga de la semana de trabajo contribuyendo a índice de adenomas pérdidos ${ }^{9}$. Se deberán realizar más estudios para comprobar estos hallazgos.

\section{Financiamiento}

La presente investigación no ha recibido ayudas específicas provenientes de agencias del sector público, sector comercial o entidades sin ánimo de lucro.

\section{Conflicto de intereses}

Los autores declaran no tener conflicto de intereses.

\section{Bibliografía}

1. Cash B, Allen C, Sharma P. NER1006 1 liter Polyethylene Glycol-Based Bowel Preparation Safety Profile in Patients with mild or moderate renal impairment: a pooled analysis of two phase 2 trials. Sesión de carteles presentada en: DDW; 2021 mayo 21-23; Virtual. Poster: Sa038.

2. Hong SN, Lee CK, Im JP, Choi CH, Byeon JS, Cho YS, et al. Efficacy and safety of very low-volume bowel preparation with $1 \mathrm{~L}$ Plenvu ${ }^{\circledR} \mathrm{com}$ parison with $2 \mathrm{~L}$ polyethylene glycol + ascorbate: Multicenter, randomized, endoscopist-blinded study. Gastrointest Endosc [Internet]. 2021;93(6 Suppl):AB10-AB11. Disponible en: https://www.giejournal.org/ article/S0016-5107(21)00331-X/fulltext

3. Jain A, Jain R, Nugent Z, Davidson D, Solati Z, Restall G, et al. A99 Optimizing colonoscopy procedures and reducing patient anxiety through recently developed online information resources. J Can Assoc Gastroenterol [Internet]. 2021;4(1):75-5. Disponible en: https://academic.oup.com/ jcag/article/4/Supplement $1 / 74 / 6158576 ?$ login $=$ true

4. Passi M, Rahman F, Koh C, Kumar S. Effects of increased patient body mass index on colonoscopy quality metrics: results from US Multi-center endoscopy outcomes database. Sesión de carteles presentada en: DDW; 2021 mayo 21-23; Virtual. Póster: Sa034.

5. Yohannan B, Bader N, Grossen A, Khan M, Nadeem M, Gupta S, et al. Predictors of increased intraluminal bubble presence during colonoscopy. Gastrointest Endosc [Internet]. 2021;93(6 Suppl):AB58. Disponible en: https://www.giejournal.org/article/S0016-5107(21)00414-4/pdf

6. Fung $P$, Syed A, et al. Poor bowel prep: are you really goinf to come back within a year? Sesión de carteles presentada en: DDW; 2021 mayo 21-23; Virtual. Póster: Fr041.

7. Sun C, Li D, Zenteno AC, et al. Low volume bowel preparation is associated with reduced time to colonoscopy in hospitalized patients: a propensity matched analysis. Sesión de carteles presentada en: DDW; 2021 mayo 21-23; Virtual. Póster: Fr053.

8. Hindi Z, Guizzetti L, Cocco S, Brahmania M, Wilson A, Yan B, et al. No evidence of a Friday effect on colonoscopy quality outcomes. J Can Assoc Gastroenterol [Internet]. 2021;4(1 Suppl):101-2. Disponible en: https://academic.oup.com/jcag/article/4/Supplement_1/101/6158659

9. Rebhun J, Yellen M, Xia Y, Shuja A. Fr054 Do gastroenterologist have a "weak" day? Impact of day of the week on outpatient screening colonoscopies. Gastroenterology. 2021;160(6):S198-S199. 